\title{
Quantification of the Influence of Particle Voids on PIV Measurements via Synthetic-PIV
}

\author{
Vincent Domogalla ${ }^{(凶)}$ \\ German Aerospace Center (DLR), Göttingen, Germany \\ vincent.domogalla@dlr.de
}

\begin{abstract}
Particle Image Velocimetry (PIV) is an important technique to investigate complex flow fields but limited to the tracking fidelity of the flow tracers and the uncertainties in the evaluation process. To examine these issues this work implements a physic-based motion simulation for small spherical particles, serving as flow tracers in PIV, with existing tools for image generation. Particular focus set on the behavior in vortical structures and the occurrence of "particle voids" due to centrifugal forces. The results show that a realistic particle distribution ideally can be achieved when applying a statistical distribution of the particle size. Furthermore, they suggest a significant error in the velocity prediction if the region of interest has no sufficient particle seeding.
\end{abstract}

Keywords: Particle Image Velocimetry $\cdot$ Vortex $\cdot$ Synthetic $\cdot$ Particle force $\cdot$ Particle dynamics $\cdot$ Helicopter $\cdot$ Blade tip $\cdot$ Error $\cdot$ Uncertainty

\section{Introduction}

Vortex structures are part of the basic phenomena in flow technology and often have a significant influence on the aerodynamic performance of aircraft, e.g. helicopters. When maneuvering, the vortex system of the main rotor can interact with the main rotor itself, the tail, and also the nacelle. The unsteady character of such vortex systems influences aerodynamic performance significantly, as well as acoustics and vibration. For a better understanding the flow field is investigated by computational simulation and wind tunnel experiments, for example by Goerttler et al. [3], Braukmann et al. [1], and Wolf et al. [14] in the rotor test facility of the DLR, German Aerospace Center in Göttingen. In these investigations, vortex development and aging are of particular interest and often examined with optical measurement techniques.

A common technique to measure flow velocities in wind tunnel experiments is Particle Image Velocimetry (PIV). Since the limitations of algorithms utilized for the evaluation of PIV experiments can influence the results significantly, this is a severe source of error and uncertainty. Heavily affected are regions with high rotational velocities and low particle density such as vortex cores. The centrifugal forces diminish the number of tracer particles resulting in poorly resolved data,

(C) The Author(s), under exclusive license to Springer Nature Switzerland AG 2021

A. Dillmann et al. (Eds.): STAB/DGLR Symposium 2020, NNFM 151, pp. 325-334, 2021.

https://doi.org/10.1007/978-3-030-79561-0_31 
named particle voids. An example of those voids is depicted in Fig. 1, showing an exemplary particle image of a PIV experiment from Wolf et al. [14] with voids at several vortex ages $\Psi$. The red circle marks the vortex position for $\Psi=325^{\circ}$ where the void has diminished due to turbulent mixing.

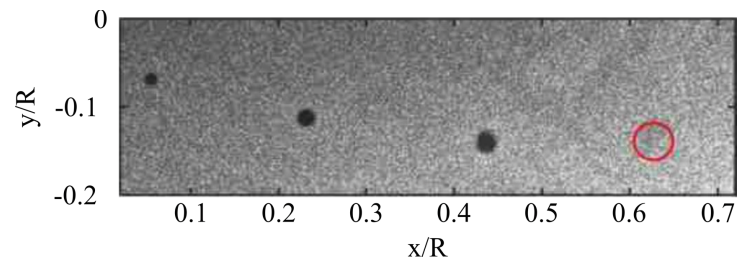

Fig. 1. Particle voids for vortex age from $\Psi=55^{\circ}$ to $235^{\circ}, \Psi=325^{\circ}$ not visible (red circle), published by Wolf et al. [14]

To validate the evaluation results, the Synthetic Particle Image Velocimetry (SPIV) was developed, not to be confused with the Stereo Particle Image Velocimetry as mentioned by Raffel et al. [8]. In this work, an existing tool was extended with dedicated particle physics to allow for a proper investigation of particle dynamics. The algorithm is based on a software stack previously developed at DLR [4] using the work of Kyle Lynch from Sandia National Laboratories and is implemented in MATLAB. The code of Lynch is used to calculate the optical projection of the particles, represented as voxels, from a three-dimensional volume onto a two-dimensional image plane. It allows the usage of multiple cameras and camera positions and also accounts for optical effects such as the impact of the aperture or the geometric position of the sensor planes relative to the image plane. Thereby, the toolchain is able to generate synthetic particle images that can be evaluated by standard PIV software. Furthermore, the Synthetic Image Generator (SIG) developed by Lecordier et al. [6] is used to generate synthetic calibration images for the subsequent evaluation.

\section{Theoretical Background}

\section{Blade Tip Vortex}

The pressure difference between the upper and lower surface of the blade causes the rotors lift force. Since the rotor blade has a finite length, this pressure difference equalizes at the blade tips. The high-pressure air is no longer blocked from returning into the low-pressure regions above the blade, causing flow around the blade tips and inducing a rotational motion. This flow then propagates into the wake and is known as the blade tip vortex.

Vortices can be quantified by their vorticity $\omega$ and circulation $\Gamma$. With these characteristics, it is possible to describe the radial velocity profile of a vortex. One of many simplified models for a vortex flow is the Lamb-Oseen vortex. It 
is derived from the Navier-Stokes equations and can be expressed depending on the radius $r$ and the time $t$ as

$$
\omega(r, t)=\frac{\Gamma_{0}}{4 \pi \nu r t}\left(1-e^{\frac{-r^{2}}{4 \nu t}}\right)
$$

with the kinematic viscosity $\nu$. The equation can be rearranged as an expression of the tangential velocity $[2,9]$ to

$$
v_{\text {tan }}(r)=v_{\text {tan, } \max }\left(1+\frac{1}{2 \alpha}\right) \frac{r_{c}}{r}\left[1-\exp \left(-\alpha\left(\frac{r}{r_{c}}\right)^{2}\right)\right] .
$$

In this case $v_{t a n}$ is the tangential velocity, $r_{c}$ the core radius and $\alpha=1.25643$ the Lamb constant.

\section{PIV}

The particle image velocimetry (PIV) is an often-used technique for the acquisition of flow data from wind-tunnel experiments. The flow field is seeded with small particles that are able to follow even small flow structures. Mostly oil droplets out of Di-Ethyl-Hexyl-Sebacat (DEHS) or vegetable oil, or Helium Filled Soap Bubbles (HFSB) are used for this purpose. To capture the flow field for quantification, the particles are illuminated with a laser, spanning a thin sheet of light. A schematic setup is shown for example in [8]. The evaluation of images, taken at two different points in time, through cross-correlation allows the calculation of the underlying flow field. The cross-correlation splits the particle image into sub-samples and calculates the most probable displacement vector between two time points for each set of sub-samples, which translates together with the time difference between both pictures into a local velocity vector. Sufficient seeding of the flow region is necessary with correlation algorithms requiring several particles (e.g. five to seven) within each sub-sample. If this condition is not met, the results of the cross-correlation can no longer be considered valid.

\section{Particle Mechanics}

The movement of rigid particles in a homogeneous flow is explained by the law of momentum conservation. Boussinesq, Basset, and Oseen formulated the first solution for this problem, known as BBO-equation. Maxey and Riley had extended this equation in 1983 [7], and various authors had further modified it. The variant of Sommerfeld [12] for rigid particles and a homogeneous flow states as follows

$$
\begin{aligned}
m_{p} \frac{d \mathbf{v}_{\mathbf{p}}}{d t}= & \frac{18 \eta_{f}}{\rho_{p} d_{p}^{2}} m_{p}\left(\mathbf{u}-\mathbf{v}_{\mathbf{p}}\right)-m_{f} \frac{D \mathbf{u}}{D t}+\frac{1}{2} m_{f}\left(\frac{D \mathbf{u}}{D t}-\frac{d \mathbf{v}_{\mathbf{p}}}{d t}\right)+\left(m_{p}-m_{f}\right) \mathbf{g} \\
& +9 \sqrt{\frac{\rho_{f} \eta_{f}}{\pi}} \frac{m_{p}}{\rho_{p} d_{p}} \int_{0}^{t} \frac{\frac{D \mathbf{u}}{D \tau}-\frac{d \mathbf{v}_{\mathbf{p}}}{d \tau}}{\sqrt{t-\tau}} d \tau
\end{aligned}
$$


where $m_{p}$ is the particle mass, $m_{f}$ the mass of the surrounding fluid displaced by the particle, $\mathbf{v}_{\mathbf{p}}$ the particle velocity, and $\mathbf{u}$ the velocity of the fluid. These velocities are depending on the position of the particle $x_{p}$ at the time $t$. Furthermore, $d_{p}$ describes the particle diameter, $\eta_{f}$ the dynamic viscosity of the fluid, $\rho_{f}$ its density, $\rho_{p}$ the particle density and $\mathbf{g}$ is the gravitational vector. The different terms can be understood as the contribution of different forces, terms on the right side of the equation:

1. Drag force $F_{D}$

2. Pressure force $F_{p}$

3. Virtual Mass Force $F_{V M}$

4. Hydro-static lift force $F_{L}$

5. Basset force $F_{B}$

Further modifications to Eq. 3 and additional forces are also discussed by Sommerfeld [12]. Due to the forces acting on the particle, its response time to a spontaneous acceleration is limited by its mass and inertia, also explaining the reason for particle voids. Since the particle cannot react quickly enough to the change in flow direction within the vortex, it gets carried out of the core. The response time $\tau_{p}$ can be derived from Eq. 3 as

$$
\tau_{p}=\frac{d_{p}^{2} \rho_{p}}{18 \eta_{f}}
$$

by neglecting every force but the drag force, which dominates in the low Re regime [8].

\section{Synthetic Particle Image Velocimetry}

The Synthetic Particle Image Velocimetry allows the generation of synthetic particle images from three-dimensional velocity fields, e.g. CFD data. The particle trajectories, caused by different forces acting on the particle, are computed over time. Subsequently, the particle positions for an arbitrary point in time are projected to an image plane and saved as a picture. A set of two pictures with a small time delay, representing the PIV double acquisition, can be analyzed with conventional PIV software to recalculate the velocity field. A comparison of the original data and the recalculated velocity reveals regions of high uncertainty. These are caused by the limitations of the evaluation algorithm in regions of low particle density. In the previous work of Hartje [4], the baseline concept for the SPIV was developed and tested. It is based on the code of Kyle Lynch, which is responsible for the projection of the particles from the three-dimensional computation space onto the two-dimensional image plane and the image calculation itself. The projection is done with the pinhole camera model from Tsai [13]. In this work dedicated particle physics is added to the tool, which was neglected in the previous version. 


\section{Modification of the Equation of Motion}

To avoid unnecessary computation time and complexity, Eq. 3 was modified by removing negligible forces from the equation, in particular the Basset force, which is only relevant for density rations $\rho_{p} / \rho_{f} \approx 1$. Furthermore, Saffmann and Magnus forces, as mentioned by Sommerfeld [12], are negligible because of the small particle diameter. Their influence is estimated by the equations given by Sommerfeld [11]. Other forces such as the Coulomb force or the thermophoretic force are insignificant for the considered case, leaving only the drag $F_{D}$, pressure $F_{P}$, and virtual mass force $F_{V M}$ as well as gravitational force $F_{G}$. To allow a wider range of applications, possible rarefaction effects are considered. The particle drag was modified with the Cunningham correction [12] depending on the Knudsen number $K n$. Also, the influence of the particle Reynolds number $R e_{p}$ had to be accounted for, using the correction of Schiller and Nauman [10]. Combining both terms in the factor $K$, which is

$$
K= \begin{cases}\left(1+K n\left[2.514+0.8 \cdot \exp \left\{\frac{-0.55}{K n}\right\}\right]\right)^{-1} & 0.1<K n<1000 \& R e_{p}<0.25 \\ 1 & K n<0.1 \& R e_{p} \leq 1 \\ 1+0.15 \cdot \operatorname{Re}_{p}^{0.687} & K n<0.1 \& R e_{p}>1,\end{cases}
$$

gives the final equation of motion as

$$
\begin{aligned}
\frac{d \mathbf{v}_{\mathbf{p}}}{d t}= & 18 \frac{\rho_{f}}{\rho_{p}} \frac{K}{d_{p}^{2}} \nu_{f}\left(\mathbf{u}-\mathbf{v}_{\mathbf{p}}\right)+\frac{\rho_{f}}{\rho_{p}}((\mathbf{u} \cdot \nabla) \mathbf{u}-\mathbf{g}) \\
& +\frac{1}{2} \frac{\rho_{f}}{\rho_{p}}\left((\mathbf{u} \cdot \nabla) \mathbf{u}-\frac{d \mathbf{v}_{\mathbf{p}}}{d t}\right)+\mathbf{g}
\end{aligned}
$$

where $\nu_{f}$ is the kinematic viscosity of the fluid and $\nabla$ is the nabla operator.

\section{Implementation}

The computation of the equation of motion is implemented following a Lagrangian approach, allowing for continuous tracking of the particles over time. After initializing the flow field, which could either be an artificial case such as a Lamb-Oseen vortex or genuine data from CFD simulation or measurement, a certain number of particles are placed at random positions, ensuring a sufficient particle density after Keane and Adrian [5]. Afterward, the trajectories are computed over time by solving Eq. 6. Transforming the equation from a differential equation of second order to a system of first-order equations allows the usage of the ode 45 differential equation solver of MATLAB with an adaptive timestep to derive the solution. After the simulation is finished, a set of images with the current particle positions is generated for every desired time step. To ensure sufficient seeding, the number of particles within the computed area must be constant. Therefore, the particles exceeding the boundaries of the computed area are replaced with new particles at random positions within the volume. This phenomenon also occurs in real PIV experiments, when tracer particles leaving the light sheet perpendicular to the plane and is known as out of plane loss. 


\section{Test Case}

To verify the results of the particle simulation a generic Lamb-Oseen vortex was used as a reference flow field derived from Eq. 2. The core radius $r_{c}$ was chosen to $5.4 \mathrm{~mm}$ and the max. tangential velocity $v_{\text {tan.max }}$ to $29.5 \mathrm{~m} / \mathrm{s}$. The vortex parameters are chosen to fit the measurement data from Wolf [14]. Additional parameters are listed in Table 1a. A parameter study was conducted to evaluate the influence of the tracer particle diameter, simulating several unique values and statistical size distributions, as suggested by Raffel et al. [8]. A log-normal distribution with the probability density function (PDF)

$$
f_{m}\left(d_{p}\right)=\frac{1}{\sqrt{2 \pi} d_{p} \cdot \sigma_{G}} \cdot \exp \left[-\frac{1}{2}\left(\frac{\ln \left(d_{p}\right)-\mu}{\sigma_{G}}\right)^{2}\right]
$$

is fitted to the data from Raffel et al. [8] with the particle diameter $d_{p}$ as random variable, giving $\mu=0.17$ and $\sigma_{G}=0.71$. As baseline data, the measurement of the q3-distribution of DEHS particles with a calibrated nozzle at 1 bar was used. Other size distributions (e.g. q1 and q2) were not considered, because the particles are assumed to be a point mass with a constant density. In Fig. 2 a comparison of the curve fit and the measurement data is depicted exemplary for $d_{p}=1 \mu \mathrm{m}$. The hatched area marks the particle sizes used for the simulation. Smaller particles were not considered because of missing verification data and the decreasing visibility of the particles, as well as particles larger than $6.5 \mu \mathrm{m}$, which are too heavy and would fall to the ground immediately. Important material parameters are listed in Table $1 b$.

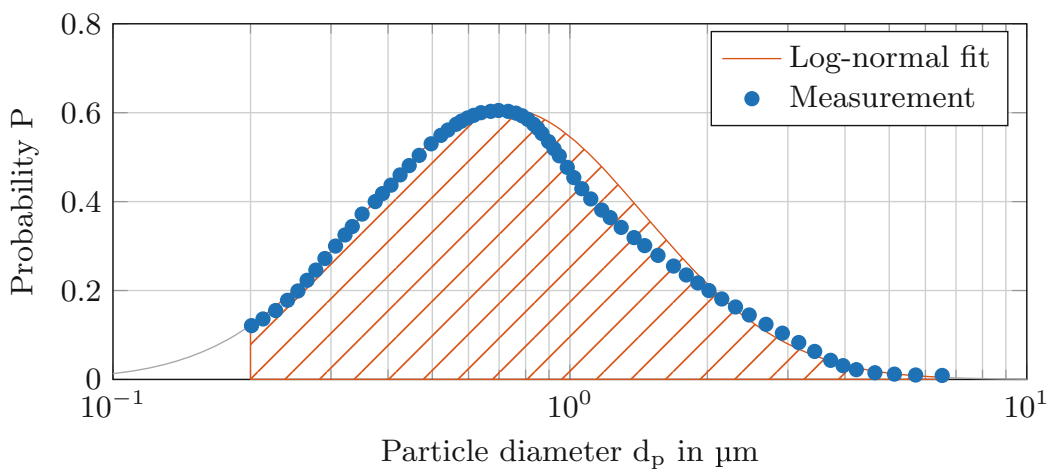

Fig. 2. Comparison of the fitted PDF of the particle diameter with the measurement data from Raffel et al. [8] 
Table 1. Overview of the parameters used for simulation and evaluation

(a) PIV parameters

\begin{tabular}{|c|c|c|c|c|c|c|c|}
\hline \multicolumn{4}{|c|}{ Simulation Setup } & \multicolumn{4}{|l|}{ Fluid: Air } \\
\hline Time delay & $\triangle \mathrm{t}$ & 25 & $\mu \mathrm{s}$ & Temperature & $\theta_{\mathrm{f}}$ & 20 & ${ }^{\circ} \mathrm{C}$ \\
\hline Sensor plane & & $16.6 \times 14$ & $\mathrm{~mm}^{2}$ & Pressure & $\mathrm{p}_{\mathrm{f}}$ & 1013 & mbar \\
\hline Pixel size & & 6.5 & $\mu \mathrm{m}$ & Density & $\rho_{\mathrm{f}}$ & 1.205 & $\mathrm{~kg} \mathrm{~m}^{-3}$ \\
\hline Timstep & & adaptive & - & Dynamic viscosity & $\eta_{\mathrm{f}}$ & $18 \times 10^{-6}$ & Pas \\
\hline Aperture & $\mathrm{f}$ & 50 & $\mathrm{~mm}$ & Kinematic viscosity & $\nu_{\mathrm{f}}$ & $15 \times 10^{-5}$ & $\mathrm{~m}^{2} \mathrm{~s}^{-1}$ \\
\hline Object distance & G & 1500 & $\mathrm{~mm}$ & Kolmogorov length & $\eta_{\mathrm{K}}$ & 4 & $\mu \mathrm{m}$ \\
\hline \multicolumn{4}{|c|}{ Evaluation Parameters } & \multicolumn{4}{|l|}{ Particles: DEHS } \\
\hline Windowsize & & 16 & $\mathrm{px}$ & Temperature & $\theta$ & 20 & ${ }^{\circ} \mathrm{C}$ \\
\hline Overlap & & 75 & $\%$ & Density & $\rho_{\mathrm{p}}$ & 912 & $\mathrm{~kg} \mathrm{~m}^{-3}$ \\
\hline
\end{tabular}

\section{Results}

Examples of the generated particle images can be found in Fig. 3. The images show the particle void developed for different particle diameters at a vortex age of $\Psi=55^{\circ}$ (corresponding to a time of $t=6.47 \mathrm{~ms}$ with a blade frequency of $f=23.6 \mathrm{~Hz}$ ). From the left to the right, in the first two images (a, b) monodisperse particles with a diameter of $1 \mu \mathrm{m}$ or $3 \mu \mathrm{m}$ was chosen, respectively. For the following three pictures (c-e), a non-uniform size distribution was applied as proposed by Raffel et al. [8] and stated in Eq. 7. The corresponding (mean) diameter of the particles $d_{p}\left(d_{p, m}\right)$ is given below the images. Monodisperse particles are leading to a rapid increase of the void size, especially for young vortex ages and large particles $(3 \mu \mathrm{m})$. A non-uniform size distribution results in a much slower and more realistic void development, especially for older vortices. This behavior can be explained by the particle mass and inertia. Increasing the size also increases the mass and inertia, which leads to a slow particle response (see Eq.4). In the case of the non-uniform size distribution, many small particles remain within the vortex resulting in a much more diffuse void boundary. The analysis of the void size and the following comparison to measurement data is pictured in Fig. 4 . The void radius, $r_{v o i d}$, was determined using the intensity

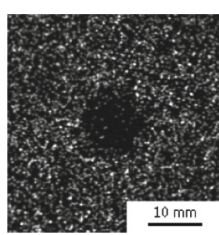

(a) $d_{p}=1 \mu m$

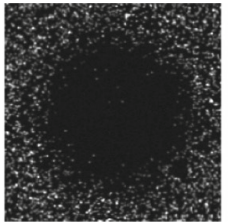

(b) $d_{p}=3 \mu \mathrm{m}$

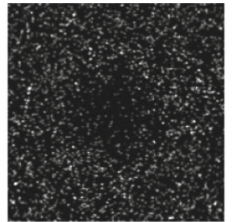

(c) $d_{p, m}=1.18 \mu \mathrm{m}$

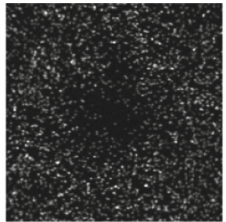

(d) $d_{p, m}=1.48 \mu \mathrm{m}$

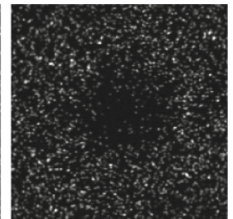

(e) $d_{p, m}=1.78 \mu \mathrm{m}$

Fig. 3. Particle voids for different particles diameter at a vortex age of $\Psi=55^{\circ}$ showing the influence of a non-uniform particle size on the void development. 
distributions of the SPIV images. Applying a spatial low-pass filter removes individual particles and enables to differentiate between seeded and non-seeded areas using an appropriate threshold. The figure shows that the usage of a statistical particle size distribution in comparison to a monodisperse particle size leads to a better agreement with the measurement data when considering the void size. Nevertheless, the constant particle diameter of $1 \mu \mathrm{m}$ shows a reasonable replication of the measurement data for a vortex age up to $\Psi=55^{\circ}$. For older vortices the influence of smaller particles remaining close to the vortex core increases and the void size grows faster than the measurement data suggests. Since the computation time and memory requirements for smaller particles increases dramatically, the monodisperse approach with a diameter of $1 \mu \mathrm{m}$ is favorable for younger vortices.

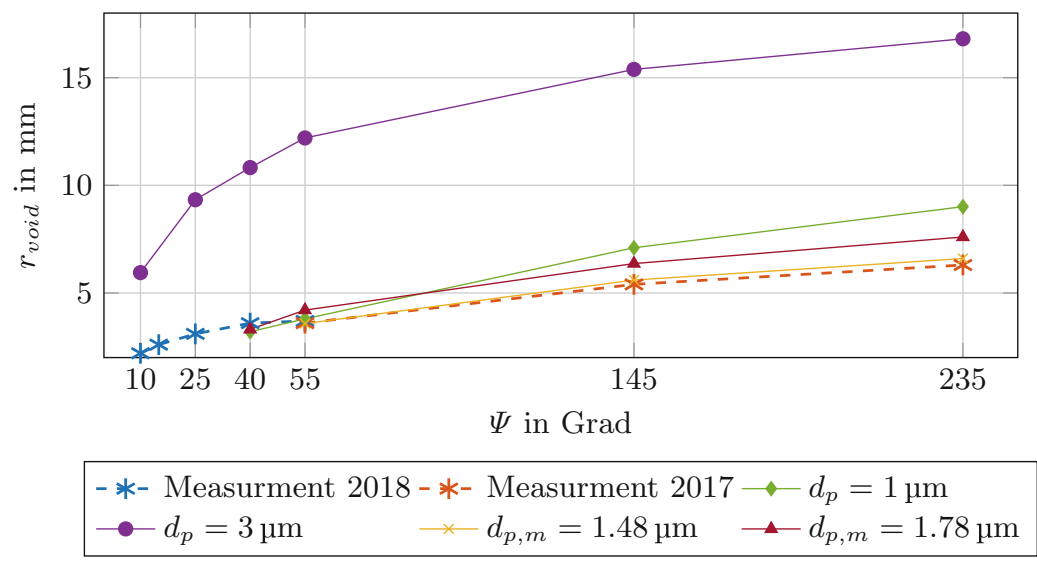

Fig. 4. Development of the void size with measurement data for different particle diameters

In Fig. 5 the velocity distribution for a constant particle diameter of $1 \mu \mathrm{m}$ (yellow line) and $3 \mu \mathrm{m}$ (orange line) are compared to the reference velocity distribution (blue line). For the sake of computation time, the monodisperse approach was chosen. The velocities are derived from the generated particle images by the commercial PIV software LaVision DaVis 8.2. The data shows a good agreement between the input reference velocity (blue line) and the SPIV evaluation even within the particle void (purple line). If the particle size exceeds a certain diameter, the velocity profile matches only outside of the particle void (green line). The low particle density within the void leads to a low or even zero signal-tonoise ratio and a high number of outliers. These outliers are detected by the PIV algorithm and replaced with a linear interpolation of the local velocity used as an initial value for subsequent steps in the multi-pass processing. For a particle diameter of $1 \mu \mathrm{m}$, this circumstance does not affect the results much because the velocity profile in the void can be considered linear. For larger particles and larger voids, the linear approximation results in a significant error because of the nonlinear velocity profile in the void. 


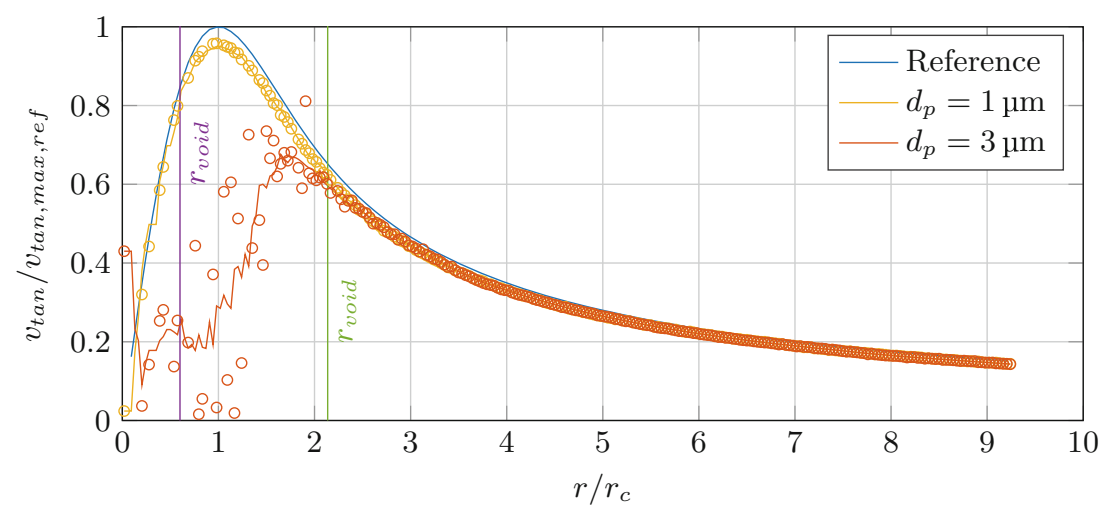

Fig. 5. Relative tangential velocity for a constant particle diameter for a vortex age of $\Psi=55^{\circ}$ with $v_{t a n, \max }=29,5 \mathrm{~m} / \mathrm{s}$ and $r_{c}=5,4 \mathrm{~mm}$, void size $1 \mu \mathrm{m}$ : purple, $3 \mu \mathrm{m}$ : green

\section{Conclusion}

This work describes the development of a tool for the creation of synthetic PIV images and presents results of its application. The tool allows for a physically correct calculation of particle trajectories based on an adapted Maxey-Riley equation (Eq. 6). From the calculated particle positions, synthetic particle images are generated. To verify the results, a generic velocity field of a Lamb-Oseen vortex based on measurement data was used.

The validation of the synthetic PIV images showed an overall good agreement with the measurement data. The variation of the particle size revealed a significant influence of the particle diameter on the results. A non-uniform size distribution with a mean diameter $d_{p, m}=1.48 \mu \mathrm{m}$ was found to match the measurement data best. However, the assumption of a constant diameter of $1 \mu \mathrm{m}$ showed reasonable results for vortex ages up to $\Psi=55^{\circ}$, while saving computational resources. The voids size matches the measurement data (Fig. 4) and the velocity profile in Fig. 5 is reproduced good enough for further investigations. This work also shows that it is not possible to interpolate sparse velocity data within the particle void if the void exceeds the vortex core radius, as seen in Fig. 5 .

Acknowledgements. The work presented in this paper is part of my master thesis "Quantitative Erfassung von Wirbeln durch Impfpartikel und bildgebende Messtechniken" (Leibniz University Hannover, 2019), which contains additional aspects and investigations. The author wants to thank Kyle Lynch for providing the source code for the particle projection and image calculation on which this work is based. 


\section{References}

1. Braukmann, J.N., Wolf, C.C., Goerttler, A., Raffel, M.: Blade tip vortex system of a rotor with cyclic pitch. AIAA J. 58(7), 2869-2880 (2020). https://doi.org/10. 2514/1.J058678

2. Breitsamter, C.: Nachlaufwirbelsysteme großer Transportflugzeuge: Experimentelle Charakterisierung und Beeinflussung. Aerodynamik. Utz, München (2007)

3. Goerttler, A., Braukmann, J.N., Wolf, C.C., Gardner, A.D., Raffel, M.: Blade tipvortices of a four-bladed rotor with axial inflow. Vertical Flight Society 75 th Annual Forum \& Technology Display, vol. 75. Vertical Flight Society (2019). https://doi. org/10.4050/JAHS.65.042002

4. Hartje, L.: Untersuchung des Einflusses der Particle Image Velocimetry auf die Charakterisierung von Blattspitzenwirbeln. Master thesis, Leibniz Unisersität Hannover (2018)

5. Keane, R.D., Adrian, R.J.: Optimization of particle image velocimeters. I. Double pulsed systems. Measur. Sci. Technol. 1(11), 1202 (1990). https://doi.org/10.1088/ 0957-0233/1/11/013

6. Lecordier, B., Demare, D., Vervisch, L.M.J., Réveillon, J., Trinité, M.: Estimation of the accuracy of PIV treatments for turbulent flow studies by direct numerical simulation of multi-phase flow. Measure. Sci. Technol. 12(9), 1382 (2001). https:// doi.org/10.1088/0957-0233/12/9/302

7. Maxey, M.R.: Equation of motion for a small rigid sphere in a nonuniform flow. Phys. Fluids 26(4), 883 (1983). https://doi.org/10.1063/1.864230

8. Raffel, M., Willert, C.E., Scarano, F., Kähler, C.J., Wereley, S.T., Kompenhans, J.: Particle Image Velocimetry. Springer, Cham (2018). https://doi.org/10.1007/ 978-3-319-68852-7

9. Saffman, P.G.: Vortex Dynamics. Cambridge Monographs on Mechanics. Cambridge University Press, West Nyack (2012)

10. Schiller, L., Nauman, A.: Über die grundlegenden Berechnungen bei der Schwerkraftaufbereitung. Zeitschrift des VDI 77, 318-320 (1933)

11. Sommerfeld, M.: Modellierung und numerische Berechnung von partikelbeladenen turbulenten Strömungen mit Hilfe des Euler-Lagrange-Verfahrens: Zugl.: Erlangen, Nürnberg, Univ., Habil.-Schr., 1996. Berichte aus der Strömungstechnik. Shaker, Aachen (1996)

12. Sommerfeld, M.: Bewegung fester Partikel in Gasen und Flüssigkeiten. In: Stephan, P., Mewes, D., Kind, M., Schaber, K., Wetzel, T., Kabelac, S. (eds.) VDIWärmeatlas, Springer Reference Technik. Springer, Berlin (2018). https://doi.org/ 10.1007/978-3-662-52991-1_88-2

13. Tsai, R.: A versatile camera calibration technique for high-accuracy 3D machine vision metrology using off-the-shelf TV cameras and lenses. IEEE J. Robot. Autom. 3(4), 323-344 (1987). https://doi.org/10.1109/JRA.1987.1087109

14. Wolf, C.C., Braukmann, J.N., Stauber, W., Schwermer, T., Raffel, M.: The tipvortex system of a four-bladed rotor in dynamic stall conditions. In: American Helicopter Society 74th Annual Forum (2018) 